\title{
Properties of ${ }^{12} \mathrm{Be}$ and ${ }^{11} \mathrm{Be}$ in terms of single-particle motion in deformed potential
}

\author{
I. Hamamoto ${ }^{1,2}$ and S. Shimoura ${ }^{3}$ \\ 1 Division of Mathematical Physics, \\ Lund Institute of Technology at the University of Lund, \\ Lund, Sweden \\ 2 The Niels Bohr Institute, \\ Blegdamsvej 17, Copenhagen Ø, \\ DK-2100, Denmark \\ 3 Center for Nuclear Study, \\ University of Tokyo, \\ Saitama 351-0198, Japan
}

\begin{abstract}
Inspired by the recent measurement of the $\mathrm{B}\left(\mathrm{E} 2 ; 0_{2}^{+} \rightarrow 2_{1}^{+}\right)$and $\mathrm{B}\left(\mathrm{E} 0 ; 0_{2}^{+} \rightarrow 0_{1}^{+}\right)$values in ${ }^{12} \mathrm{Be}$, we give an interpretation of available spectroscopic data on both ${ }^{12} \mathrm{Be}$ and ${ }^{11} \mathrm{Be}$, using a simple model which contains the essential feature of these two nuclei; the presence of weakly-bound neutron(s) in deformed potentials. The agreement of the calculated results with observed data is surprisingly good, including well-known strong E1 transitions in both nuclei.
\end{abstract}

PACS numbers: 21.60.Ev, 21.10.Ky, 21.10.Pc, 27.20.+n 


\section{INTRODUCTION}

The study of the properties of nuclei far from the line of $\beta$ stability is currently one of the most active and challenging topics in nuclear structure. Exotic ratios of $\mathrm{Z} / \mathrm{N}$ for a given mass number together with the presence of weakly bound nucleons lead to the phenomena which are unexpected from our common sense about stable nuclei; among others, one finds the change of the shell structure and magic numbers. Observed properties of low-lying states in ${ }_{4}^{11} \mathrm{Be}_{7}$ and ${ }_{4}^{12} \mathrm{Be}_{8}$ have contributed to the finding that $\mathrm{N}=8$ is no longer a magic number in such unstable nuclei.

Though there have been already many elaborate attempts to describe the structure of Be isotopes, in the present paper we give an interpretation of available spectroscopic data on ${ }^{12} \mathrm{Be}$ and ${ }^{11} \mathrm{Be}$ keeping a model as simple as possible, while taking duly into account the essential feature of these nuclei; the presence of weakly-bound neutron(s) in finite deformed potentials. A simplest version of Bohr-Mottelson model (see Chaps. 4 and 5 of Ref. [1]) is applied to the nuclei ${ }^{11} \mathrm{Be}$ and ${ }^{12} \mathrm{Be}$, using weakly-bound one-particle wave-functions estimated properly with deformed Woods-Saxon potentials instead of harmonic-oscillator potentials. The present work is prompted by the recent lifetime measurements of the $0_{2}^{+}$ state in ${ }^{12} \mathrm{Be}[2]$.

The deformation of Be isotopes has been studied using various theoretical models; for example, ${ }^{11} \mathrm{Be}$ is studied using models with deformed Woods-Saxon potentials [3, 4], ${ }^{11} \mathrm{Be}$ based on the Nilsson-Strutinsky method [5], and neutron-rich Be isotopes using the deformed Hartree-Fock calculation with Skyrme interactions [6]. The models used in Refs. [3, 4], in which the idea of the particle-rotor model is used, may be closest to our present model. However, since in very light nuclei such as Be isotopes the nuclear shape may be considerably changed just by adding one neutron, we would avoid to use the observed properties of the

core nucleus ${ }^{10} \mathrm{Be}$, in contrast to the work of Refs. [3, 4]. Since only low-energy states are discussed in the present work, we assume that four protons and six neutrons are treated as being inactive occupying the lowest possible Nilsson levels for the same deformation as that for the extra neutron(s). Thus, the inactive core is certainly different from the actual ${ }^{10} \mathrm{Be}$ nucleus.

Moreover, the rotational perturbation of the intrinsic nuclear structure is neglected since only those states close to the band head are studied in the present work. Thus, we try to see 
how the observed properties of low-lying levels of ${ }^{11} \mathrm{Be}$ and ${ }^{12} \mathrm{Be}$ can be understood in terms of weakly-bound neutron(s) in deformed potentials. Deformed nuclear halos are studied in

Ref. [7] using a finite square-well potential without spin-orbit term, while in Ref. [8] they are investigated with more realistic potentials: deformed Woods-Saxon potentials with a spin-orbit term.

In Sec. II some aspects of the model and formulas are summarized. In Sec.III numerical results are presented and discussed in comparison with experimental data, while in Sec. IV conclusions are given.

\section{MODEL AND FORMULAS}

It is known [1] that in medium-heavy deformed nuclei the analysis of low-energy spectroscopic properties in terms of one-particle motions in a deformed potential works impressively well, especially if the pair-correlation in the form of BCS quasiparticles is included. This is because the major part of the long-range two-body interaction is already taken into account in the deformed mean-field. In very light nuclei such as Be isotopes the many-body pair correlation that originates from a short-range interaction can be negligible. Therefore, in the present work we try to describe the spectroscopic properties of low-lying states of ${ }^{11} \mathrm{Be}$ and ${ }^{12} \mathrm{Be}$ in terms of two Nilsson levels occupied by the seventh neutron and the seventh and eighth neutrons, respectively, in deformed Woods-Saxon potentials. The pairing interaction between two weakly-bound neutrons in ${ }^{12} \mathrm{Be}$ will be duly taken into account. This simple description may be justified, since there are no nearby Nilsson levels in the region of deformation, where the $[220,1 / 2]$ and $[101,1 / 2]$ levels are almost degenerate.

One-particle levels with quantum numbers $\mathrm{K}^{\pi}$ in a $Y_{20}$-deformed potential (Nilsson levels) are denoted by the asymptotic quantum numbers, $\left[N, n_{z}, \Lambda, \Omega\right]$, where $\Omega=K$ and $\pi=$ $(-1)^{N}$. The asymptotic quantum numbers express, in a good approximation, the wave functions of one-particle levels well bound in sufficiently deformed potentials. When a oneparticle level becomes weakly bound, the wave function can be very different from that indicated by the asymptotic quantum numbers, even when the one-particle potential is well deformed [8]. Nevertheless, just for convenience sake, in the present work we use the asymptotic quantum numbers to denote respective Nilsson levels, also in the case that those levels become weakly bound. 
In the leading order the rotational energy spectrum is written as

$$
E_{\text {rot }}=\frac{\hbar^{2}}{2 \Im}\left(I(I+1)+a(-1)^{I+1 / 2}\left(I+\frac{1}{2}\right) \delta(K, 1 / 2)\right)
$$

where $\Im$ expresses the moment of inertia while the decoupling parameter is denoted by a. The expression (11), in which the rotational perturbation of the intrinsic structure is neglected, is expected to work well for low-lying rotational states close to the band head.

The formulas for $\mathrm{B}(\mathrm{E} \lambda)$ to be used in the present work are (see Eqs. (4-91) and (4-92) of Ref. [1])

$$
\begin{aligned}
B\left(E \lambda ; K_{1} I_{1} \rightarrow K_{2} I_{2}\right)= & \left\{C\left(I_{1} \lambda I_{2} ; K_{1}, K_{2}-K_{1}, K_{2}\right)\left\langle K_{2}\left|E\left(\lambda, \mu=K_{2}-K_{1}\right)\right| K_{1}\right\rangle\right. \\
+ & \left.(-1)^{I_{1}+K_{1}} C\left(I_{1} \lambda I_{2} ;-K_{1}, K_{1}+K_{2}, K_{2}\right)\left\langle K_{2}\left|E\left(\lambda, \mu=K_{1}+K_{2}\right)\right| \tilde{K}_{1}\right\rangle\right\}^{2} \\
& \text { for } K_{1} \neq 0 \text { and } K_{2} \neq 0
\end{aligned}
$$

and

$$
\begin{aligned}
B\left(E \lambda ; K_{1}=0, I_{1} \rightarrow K_{2} I_{2}\right)= & \left\{C\left(I_{1} \lambda I_{2} ; 0 K_{2} K_{2}\right)\left\langle K_{2}\left|E\left(\lambda, \mu=K_{2}\right)\right| K_{1}=0\right\rangle\right\}^{2} \\
& \left\{\begin{array}{lll}
2 & \text { for } & K_{2} \neq 0 \\
1 & \text { for } & K_{2}=0
\end{array}\right.
\end{aligned}
$$

where intrinsic matrix-elements are expressed by $\left\langle K_{2}|E(\lambda, \mu)| K_{1}\right\rangle$.

From two different pairs of one-particle orbits $\left(\nu_{1} \tilde{\nu_{1}}\right)$ and $\left(\nu_{2} \tilde{\nu_{2}}\right)$ in deformed potentials, where $\tilde{\nu}$ expresses the time-reversed orbit of $\nu$, one can form two orthogonal two-particle configurations

$$
\begin{aligned}
& \left|K^{\pi}=0_{1}^{+}\right\rangle=\left(a^{2}+b^{2}\right)^{-1 / 2}\left(a\left|\nu_{1} \tilde{\nu_{1}}\right\rangle+b\left|\nu_{2} \tilde{\nu_{2}}\right\rangle\right) \\
& \left|K^{\pi}=0_{2}^{+}\right\rangle=\left(a^{2}+b^{2}\right)^{-1 / 2}\left(-b\left|\nu_{1} \tilde{\nu_{1}}\right\rangle+a\left|\nu_{2} \tilde{\nu_{2}}\right\rangle\right)
\end{aligned}
$$

This mixture of the two $|\nu \tilde{\nu}\rangle$ configurations can be interpreted as the result of pairing interaction. The quadrupole matrix element connecting these two states in the intrinsic system is written as

$$
\left\langle K^{\pi}=0_{2}^{+}\left|\sum_{k}\left(r^{2} Y_{20}\right)_{k}\right| K^{\pi}=0_{1}^{+}\right\rangle=\frac{2 a b}{a^{2}+b^{2}}\left(\left\langle\nu_{2}\left|r^{2} Y_{20}\right| \nu_{2}\right\rangle-\left\langle\nu_{1}\left|r^{2} Y_{20}\right| \nu_{1}\right\rangle\right)
$$

Similarly, the monopole matrix element is

$$
\left\langle K^{\pi}=0_{2}^{+}\left|\sum_{k}\left(r^{2}\right)_{k}\right| K^{\pi}=0_{1}^{+}\right\rangle=\frac{2 a b}{a^{2}+b^{2}}\left(\left\langle\nu_{2}\left|r^{2}\right| \nu_{2}\right\rangle-\left\langle\nu_{1}\left|r^{2}\right| \nu_{1}\right\rangle\right)
$$


The unique feature of these transition matrix-elements is illuminated on p.552-553 of Ref. [1], in connection with pair correlation and $\beta$ vibration.

In order to obtain the wave functions of one-particle levels in deformed Woods-Saxon potentials, the coupled-channel equations for a given one-particle level are solved [8] in coordinate space with correct asymptotic conditions for $r \rightarrow \infty$, without confining the system in a finite box.

\section{NUMERICAL RESULTS}

\section{A. Energy and deformation parameter}

In ${ }^{11}$ Be known as a one-neutron halo nucleus the presence of an extremely strong E1 transition, $B\left(E 1 ; 1 / 2^{-} \rightarrow 1 / 2^{+}\right)=(0.115 \pm 0.01) \mathrm{e}^{2} \mathrm{fm}^{2}$, is well known for years [9]. There are only two known bound states in ${ }^{11} \mathrm{Be}$ : the $1 / 2^{+}$ground state and the $1 / 2^{-}$state at $\mathrm{Ex}=0.32 \mathrm{MeV}$. Due to the very small neutron separation energy $S(n)=0.504 \mathrm{MeV}$, there is no hope to observe enhanced E2 transitions to establish possible rotational spectra based on the ground state. However, at least three resonant levels, $5 / 2^{+}$at $\mathrm{Ex}=1.78 \mathrm{MeV}, 3 / 2^{-}$at $\mathrm{Ex}=2.69 \mathrm{MeV}$ and $3 / 2^{+}$at $\mathrm{Ex}=3.41 \mathrm{MeV}$, have been established by recent experiments [10, 11]. If we regard the two one-particle resonant levels, $5 / 2^{+}$at $\mathrm{Ex}=1.78 \mathrm{MeV}$ and $3 / 2^{+}$at $\mathrm{Ex}=3.41 \mathrm{MeV}$, as members of the rotational band based on the ground state with $I^{\pi}=K^{\pi}=1 / 2^{+}$, we obtain the decoupling parameter $a=1.82$ and the rotational constant

$\hbar^{2} / 2 \Im=0.403 \mathrm{MeV}$. Then, using the expression in Eq. (1), the member of the ground-state rotational band in ${ }^{11} \mathrm{Be}$ next lowest to $3 / 2^{+}$is the $9 / 2^{+}$state, which is expected around $\mathrm{Ex}=6.7 \mathrm{MeV}$ in the absence of rotational perturbation. The centrifugal barrier for the $9 / 2^{+}$ level is quite high and, thus, the possible resonance will be relatively sharp in spite of its higher energy.

Assuming that the moment of inertia $\Im$ is proportional to $A^{5 / 3}, \hbar^{2} / 2 \Im=0.349 \mathrm{MeV}$ is obtained for ${ }^{12} \mathrm{Be}$. Then, we estimate the excitation energy of the $2_{1}^{+}$state in ${ }^{12} \mathrm{Be}$ to be

$$
E\left(2_{1}^{+}\right)=6(0.349)=2.09 \mathrm{MeV}
$$

which indeed agrees with the observed value $2.11 \mathrm{MeV}$. This agreement may indicate that the two nuclei, ${ }^{12} \mathrm{Be}$ and ${ }^{11} \mathrm{Be}$, have similar deformations. 
In ${ }^{11} \mathrm{Be}$ we assume that the $1 / 2^{+}$and $1 / 2^{-}$levels are expressed by the seventh neutron occupying one-neutron levels, [220,1/2] and [101,1/2], respectively, for a given strong prolatedeformation. Using the standard spin-orbit strength [12], radius parameter $r_{0}=1.25 \mathrm{fm}$, and a diffuseness $a=0.65 \mathrm{fm}$ (potential [a]) or $a=1.00 \mathrm{fm}$ (potential [b]), the deformation $\beta$ and the depth of the Woods-Saxon potential $V_{W S}$ are determined so as to produce $\varepsilon([220,1 / 2])=-0.5$ $\mathrm{MeV}$ and $\varepsilon([101,1 / 2])=-0.2 \mathrm{MeV}$. We obtain

$$
\begin{array}{lll}
\beta=0.73, & V_{W S}=-38.6 \mathrm{MeV} & \text { for potential [a] } \\
\beta=0.82, & V_{W S}=-33.5 \mathrm{MeV} & \text { for potential [b] }
\end{array}
$$

The $\beta$ value determined for ${ }^{11} \mathrm{Be}$ in the literature depends on the used model and parameterization, and varies from 0.7 to 1.1 ; for example, see Ref. [4]. The diffuseness $a=0.65 \mathrm{fm}$ is a standard value for medium-heavy stable nuclei, while $a=1.00 \mathrm{fm}$ is an attempt to simulate the potential without a flat bottom that may be commonly obtained in Hartree-Fock calculations for very light nuclei. In the present work the deformed Woods-Saxon potential is needed only to obtain the wave function of the seventh neutron, though the total neutron potential in the presence of a halo neutron may not be properly described by a Woods-Saxon potential. In Table I we show the probabilities of $\ell_{j}$ components in the wave functions of the $[220,1 / 2]$ and $[101,1 / 2]$ levels estimated for the potentials [a] and [b]. It is noted that the radial wave-functions of $\ell_{j}$ components of those deformed weakly-bound levels can be considerably different from those of $n \ell_{j}$ eigenfunctions of spherical Woods-Saxon potentials [8, 13]. Therefore, great care has to be taken, when the spectroscopic factors are extracted in the analysis of the data such as the one made in Refs. [10, 11, 14]. In this sense, a caution is needed in the comparison of the $s_{1 / 2}$ probability, 0.62 , in the $[220,1 / 2]$ level calculated for the potential [b] with the spectroscopic factor obtained in Ref. [10], $0.72 \pm 0.04$.

Using the $[220,1 / 2]$ neutron wave-function for the potential [b] with $g_{R}=\mathrm{Z} / \mathrm{A}$ and $g_{s}^{e f f}=0.9 g_{s}^{\text {free }}$, we obtain $-1.72 \mu_{N}$ for the magnetic moment of the ground state of ${ }^{11} \mathrm{Be}$, which is in good agreement with the observed value, $\mu\left({ }^{11} \mathrm{Be}\right)=-1.6816(8) \mu_{N}[15]$. The calculated value is not sensitive either to the used $g_{R}$ value of the even-even core or the parameters of the Woods-Saxon potential. This insensitivity comes from the fact that the magnetic moment of an $s_{1 / 2}$ neutron is equal to $0.5 g_{s}^{e f f}$, while in the asymptotic limit the magnetic moment of the $\mathrm{I}=1 / 2$ state coming from the $[220,1 / 2]$ neutron coupled to the $\mathrm{K}=0$ core is also equal to $0.5 g_{s}^{e f f}$ independent of the core $g_{R^{-}}$value used. In other words, in the 
present $I=1 / 2$ state the value of the measured magnetic moment cannot be used to find whether or not the nucleus is deformed.

The spectroscopic properties of four known bound levels in ${ }^{12} \mathrm{Be}, 0_{1}^{+}, 2_{1}^{+}$at $\mathrm{Ex}=2.11 \mathrm{MeV}$, $0_{2}^{+}$at $\mathrm{Ex}=2.25 \mathrm{MeV}$ and $1_{1}^{-}$at $\mathrm{Ex}=2.70 \mathrm{MeV}$, have been experimentally studied for the last few years [16, 17, 18]. In particular, the recent measurement of the lifetime of the $0_{2}^{+}$level [2] has pinned down the absolute magnitudes of $B\left(E 2 ; 0_{2}^{+} \rightarrow 2_{1}^{+}\right)=(7.0 \pm 0.6) \mathrm{e}^{2} \mathrm{fm}^{2}$ and $\mid\left\langle 0_{2}^{+}\right|$e $r^{2}\left|0_{1}^{+}\right\rangle \mid=(0.87 \pm 0.03)$ e $\mathrm{fm}^{2}$, which are very valuable informations. The relatively large values of $B\left(E 2 ; 0_{2}^{+} \rightarrow 2_{1}^{+}\right)$and $\left|\left\langle 0_{2}^{+}\left|e r^{2}\right| 0_{1}^{+}\right\rangle\right|$indicate that the intrinsic configurations of the $0_{1}^{+}$and $0_{2}^{+}$states are expressed by linear combinations of two two-neutron configurations, $\left(\nu_{1} \tilde{\nu_{1}}\right)$ and $\left(\nu_{2} \tilde{\nu_{2}}\right)$, of which the intrinsic quadrupole moments are quite different. The analysis of inelastic proton scattering exciting the $2_{1}^{+}$state in inverse kinematics [16] led to the deformation length, $(2.00 \pm 0.23) \mathrm{fm}$, which suggested strong quadrupole deformation, $\beta \sim$ 0.7 .

Observed two $0^{+}$levels in ${ }^{12} \mathrm{Be}$ are assumed to be expressed by linear combinations (with equal amplitudes) of two two-neutron configurations, [220,1/2][220,1/2] and $[101,1 / 2][10 \widetilde{1,1} / 2]$, for a given prolate deformation. The relation $|a| \approx|b|$ in the expression of Eq. (4) is expected since the $[202,1 / 2]$ and $[101,1 / 2]$ levels in ${ }^{11}$ Be lie only $300 \mathrm{keV}$ apart, following the interpretation in the present model. The deformation $\beta$ and the depth of the Woods-Saxon potential $V_{W S}$ are determined so as to produce both [220,1/2] and [101,1/2] levels at $-1.1 \mathrm{MeV}$, which in the absence of the interaction between the two configurations is about a half of the binding energy of the two neutrons. Then, we obtain

$$
\begin{array}{lll}
\beta=0.66, & V_{W S}=-39.0 \mathrm{MeV} & \text { for potential [a] } \\
\beta=0.72, & V_{W S}=-35.2 \mathrm{MeV} & \text { for potential [b] }
\end{array}
$$

The intrinsic configuration assumed here for the ground state of ${ }^{12} \mathrm{Be}$ is consistent with the fact that all four states, $1 / 2^{+}, 1 / 2^{-}, 5 / 2^{+}$and $3 / 2^{-}$, of ${ }^{11}$ Be are populated in one-neutron removal reactions from ${ }^{12} \mathrm{Be}$ with comparative magnitudes of spectroscopic factors [11].

\section{B. E1 transitions}

In medium-heavy stable nuclei low-energy E1 transitions are so much hindered in both spherical and deformed nuclei that one could hardly expect to obtain a nuclear-structure 
information from observed $\mathrm{B}(\mathrm{E} 1)$ values [19]. The reason for the hindrance is: (a) no appreciable amount of low-energy E1 strength due to the nuclear shell-structure; (b) the high-lying isovector giant dipole resonance absorbs the major part of possible E1 strength; (c) isoscalar dipole mode corresponds to the center of mass motion and thus does not contribute to E1 excitations. In contrast, in nuclei with weakly bound nucleons the origin of the hindrance (a) may be removed due to the change of the shell-structure or change in the one-particle wave functions, while the hindrance (b) may be drastically reduced due to the very weak coupling of the nucleons to the well-bound core.

In well-bound and well-deformed nuclei the E1 transition between the $[220,1 / 2]$ and $[101,1 / 2]$ levels, where $\left|\Delta n_{z}\right|=2$ and $|\Delta \Lambda|=1$, is asymptotically forbidden. Therefore, if the nucleus is well deformed, the observed strong E1 transition must come from the properties of the levels related to being weakly bound. It is pointed out that the $s_{1 / 2}$ component becomes dominant in all $\Omega^{\pi}=1 / 2^{+}$Nilsson levels as the binding energy of the orbits approaches zero, though it depends on respective Nilsson levels at which binding energy the dominance occurs [8]. Since the major component of the [101,1/2] level is $p_{1 / 2}$, the $s_{1 / 2}$ dominance in the weakly-bound [220,1/2] level can produce a pretty strong E1 matrix-element. In Table II the calculated result of E1 properties is shown, where the neutron E1 effective charge, $(Z / A) e=(4 / 11) e=(0.364) \mathrm{e}$, is used neglecting the core polarization effect (namely, the effect of shifting low-energy E1 strength to the high-lying isovector giant dipole resonance) since halo neutrons may hardly polarize the well-bound core. The formula used to estimate the $\mathrm{B}(\mathrm{E} 1)$ value is

$$
\begin{aligned}
B(E 1 ; & \left.I^{\pi}=K^{\pi}=1 / 2^{+} \rightarrow I^{\pi}=K^{\pi}=1 / 2^{-}\right) \\
= & \left(e_{\text {eff }}^{n}(E 1)\right)^{2}\left\{C\left(\frac{1}{2}, 1, \frac{1}{2} ; \frac{1}{2}, 0, \frac{1}{2}\right)\left\langle[101,1 / 2]\left|r Y_{10}\right|[220,1 / 2]\right\rangle\right. \\
& \left.+(-1)^{1 / 2+1 / 2} C\left(\frac{1}{2}, 1, \frac{1}{2} ; \frac{-1}{2}, 1, \frac{1}{2}\right)\left\langle[101,1 / 2]\left|r Y_{11}\right|[220 \widetilde{1} 1 / 2]\right\rangle\right\}^{2}
\end{aligned}
$$

where $[22 \widetilde{0,1} / 2]$ expresses the time-reversed intrinsic configuration of $[220,1 / 2]$. In the present case the contribution coming from the $Y_{11}$ term to the E1 matrix element (so-called 'signature-dependent term'), which originates from the $R$ symmetry of the nuclear shape, is several times larger than that from the $Y_{10}$ term and contributes coherently. In both $Y_{10}$ and $Y_{11}$ matrix elements a smaller contribution from $d_{5 / 2} \rightarrow p_{3 / 2}$ contributes destructively to the major one from $s_{1 / 2} \rightarrow p_{1 / 2}$. The destructive structure is a remaining trace of the asymptotically forbidden E1 matrix-element. Observing that the B(E1) value obtained by 
using the potential [b] is in good agreement with the observed value, $(0.115 \pm 0.01) \mathrm{e}^{2} \mathrm{fm}^{2}$, in the following we show numerical results obtained with the potential [b].

In the $\mathrm{E} 1$ transition, $0_{1}^{+} \rightarrow 1^{-}$, of ${ }^{12} \mathrm{Be}$ the $Y_{10}$ term contributes if the $I^{\pi}=1^{-}$state has $K^{\pi}=0^{-}$, while the $Y_{11}$ term does if $K^{\pi}=1^{-}$. In both $K^{\pi}=0^{-}$and $K^{\pi}=1^{-}$cases the band head will be $I^{\pi}=1^{-}$. If $K^{\pi}=0^{-}$, the rotational band has members with $I^{\pi}=1^{-}, 3^{-}, 5^{-}, \ldots$, while the rotational band with $K^{\pi}=1^{-}$has $I^{\pi}=1^{-}, 2^{-}, 3^{-}, \ldots$ That means, if one observes the $2^{-}$rotational member among resonant levels, $K^{\pi}=1^{-}$is assigned to the $I^{\pi}=1^{-}$level at $\mathrm{Ex}=2.70 \mathrm{MeV}$. Since, to our knowledge, no resonant level that may be a candidate for the rotational member is yet found, in Table [II] we show the calculated results of both $K^{\pi}=0^{-}$ and $1^{-}$cases. The intrinsic wave functions used are

$$
\begin{aligned}
& \Phi\left(K^{\pi}=0_{1}^{+}\right)=\frac{1}{\sqrt{2}}([220,1 / 2][22 \widetilde{0,1} / 2]+[101,1 / 2][10 \widetilde{1,1} / 2]) \\
& \Phi\left(K^{\pi}=0^{-}\right)=\frac{1}{\sqrt{2}}([101,1 / 2][22 \widetilde{0,1} / 2]-[101,1 / 2][220,1 / 2]) \\
& \Phi\left(K^{\pi}=1^{-}\right)=[101,1 / 2][220,1 / 2]
\end{aligned}
$$

Then, the formulas used to estimate those $\mathrm{B}(\mathrm{E} 1)$ values are

$$
\begin{gathered}
B\left(E 1 ; I^{\pi}=K^{\pi}=0_{1}^{+} \rightarrow I^{\pi}=1^{-}, K^{\pi}=0^{-}\right)=4\left|\left\langle[101,1 / 2]\left|r Y_{10}\right|[220,1 / 2]\right\rangle\right|^{2}\left(e_{\text {eff }}^{n}(E 1)\right)^{2} \\
B\left(E 1 ; I^{\pi}=K^{\pi}=0_{1}^{+} \rightarrow I^{\pi}=K^{\pi}=1^{-}\right)=4\left|\left\langle[220,1 / 2]\left|r Y_{11}\right|[10 \widetilde{1,1} / 2]\right\rangle\right|^{2}\left(e_{\text {eff }}^{n}(E 1)\right)^{2}
\end{gathered}
$$

In Table【II $\left|e_{e f f}^{n}(E 1)\right|=(Z / A) \mathrm{e}=(4 / 12) \mathrm{e}=(0.333) \mathrm{e}$ is employed. Since the matrix-element of $r Y_{11}$ is larger than that of $r Y_{10}$, the calculated $\mathrm{B}\left(\mathrm{E} 1 ; I^{\pi}=K^{\pi}=0_{1}^{+} \rightarrow I^{\pi}=K^{\pi}=1^{-}\right)$ value is several times larger than the $\mathrm{B}\left(\mathrm{E} 1 ; I^{\pi}=K^{\pi}=0_{1}^{+} \rightarrow I^{\pi}=1^{-}, K^{\pi}=0^{-}\right)$value. Since $\left|e_{e f f}^{n}\right|$ for E1 transitions for neutrons with the binding energy of $-1.1 \mathrm{MeV}$ may be somewhat smaller than (Z/A)e due to non-negligible core-polarization effect, the assignment of $K^{\pi}=1^{-}$to the $I^{\pi}=1^{-}$state at $\mathrm{Ex}=2.70 \mathrm{MeV}$ is suggested from the comparison of the calculated and observed $\mathrm{B}(\mathrm{E} 1)$ values.

It is noted that in the present simple model, where $|a|=|b|$ is assumed in the expression (4)), both the $\mathrm{B}\left(\mathrm{E} 1 ; I^{\pi}=K^{\pi}=0_{2}^{+} \rightarrow I^{\pi}=1^{-}, K^{\pi}=0^{-}\right)$and $\mathrm{B}\left(\mathrm{E} 1 ; I^{\pi}=K^{\pi}=0_{2}^{+} \rightarrow I^{\pi}=\right.$ $K^{\pi}=1^{-}$) values in ${ }^{12} \mathrm{Be}$ vanish. Thus, it is very interesting to measure the upper limit of the E1 transition, $1^{-}$at $2.70 \mathrm{MeV} \leftrightarrow 0_{2}^{+}$at $2.25 \mathrm{MeV}$, though the small transition-energy makes the measurement very difficult. 


\section{E2 transition in ${ }^{12} \mathrm{Be}$}

Now, we consider the E2 transition, $0_{2}^{+} \rightarrow 2_{1}^{+}$, in ${ }^{12} \mathrm{Be}$. The $2_{1}^{+}$state at Ex $=2.11 \mathrm{MeV}$ is interpreted as a rotational member of the ground $0_{1}^{+}$state. Using the intrinsic configuration

$$
\Phi\left(K^{\pi}=0_{2}^{+}\right)=\frac{1}{\sqrt{2}}(-[220,1 / 2][22 \widetilde{0,1} / 2]+[101,1 / 2][10 \widetilde{1,1} / 2])
$$

and $\Phi\left(K^{\pi}=0_{1}^{+}\right)$in Eq. (8) , we obtain

$$
\begin{aligned}
B(E 2 & \left.; I^{\pi}=K^{\pi}=0_{2}^{+} \rightarrow I^{\pi}=2_{1}^{+}, K^{\pi}=0_{1}^{+}\right) \\
& =\left(e_{\text {eff }}^{n}(E 2)\right)^{2}\left\{\left\langle[220,1 / 2]\left|r^{2} Y_{20}\right|[220,1 / 2]\right\rangle-\left\langle[101,1 / 2]\left|r^{2} Y_{20}\right|[101,1 / 2]\right\rangle\right\}^{2}
\end{aligned}
$$

Using the potential [b] for ${ }^{12}$ Be we obtain

$$
\begin{gathered}
\left\langle[220,1 / 2]\left|r^{2} Y_{20}\right|[220,1 / 2]\right\rangle=11.56 \mathrm{fm}^{2} \\
\left\langle[101,1 / 2]\left|r^{2} Y_{20}\right|[101,1 / 2]\right\rangle=-0.32 \mathrm{fm}^{2}
\end{gathered}
$$

and, then,

$$
\begin{aligned}
B\left(E 2 ; I^{\pi}=K^{\pi}=0_{2}^{+} \rightarrow I^{\pi}=2_{1}^{+}, K^{\pi}=0_{1}^{+}\right) & =141\left(e_{\text {eff }}^{n}(E 2)\right)^{2} \\
& =5.6 \sim 12.7 \mathrm{e}^{2} \mathrm{fm}^{4}
\end{aligned}
$$

where $e_{e f f}^{n}(E 2)=(0.2 \sim 0.3)$ e is used in the last line. These values of $e_{\text {eff }}^{n}(E 2)$ are obtained from the analysis of measured quadrupole moments of ${ }_{5}^{15} \mathrm{~B}_{10}$ and ${ }_{5}^{17} \mathrm{~B}_{12}$ using the shell model with the $0 \hbar \omega$ space [20]. Both the large neutron excess and the weak binding of neutrons make $e_{\text {eff }}^{n}(E 2)$ smaller. The estimated value (14) is in agreement with the measured value, $\mathrm{B}\left(\mathrm{E} 2 ; 0_{2}^{+} \rightarrow 2_{1}^{+}\right)=(7.0 \pm 0.6) \mathrm{e}^{2} \mathrm{fm}^{4}$. It is noted that for the present large prolate deformation the matrix element $\left\langle[101,1 / 2]\left|r^{2} Y_{20}\right|[101,1 / 2]\right\rangle$ is positive if the $[101,1 / 2]$ level is deeply bound, in contrast to the negative value in (13). On the other hand, for a more moderate prolate deformation the matrix element is negative irrespective of one-particle energies. The dependence of the expectation values of $r^{2} Y_{20}$ in the $[101,1 / 2]$ and $[220,1 / 2]$ states on one-particle energies is exhibited in Fig. 1 for two deformation values. It is seen that in the region of $\varepsilon_{\Omega}<-1 \mathrm{MeV}$ the indication of the divergence, $\left\langle[101,1 / 2]\left|r^{2} Y_{20}\right|[101,1 / 2]\right\rangle$ $\rightarrow-\infty$ for $\left|\epsilon_{\Omega}\right| \rightarrow 0$, which comes from the behavior of $\ell=1$ one-particle wave-functions for $\left|\varepsilon_{\Omega}\right| \rightarrow 0$ [21], has not yet appeared. Thus, in the present case the calculated value

of the expression (12) is not sensitive to a small variation of $\varepsilon_{\Omega}$. The very small value of 
$\left|\left\langle[101,1 / 2]\left|r^{2} Y_{20}\right|[101,1 / 2]\right\rangle\right|$ for $\varepsilon_{\Omega}<-1 \mathrm{MeV}$ comes from the dominance of $\mathrm{p}_{1 / 2}$ component in the $[101,1 / 2]$ wave function, because the density distribution of $\mathrm{p}_{1 / 2}$ orbit is spherically symmetric. On the other hand, for all values of $\varepsilon_{\Omega}<0$ the major contribution to $\left\langle[101,1 / 2]\left|r^{2} Y_{20}\right|[101,1 / 2]\right\rangle$ comes from the $r^{2} Y_{20}$ matrix element between the components $p_{1 / 2}$ and $p_{3 / 2}$ in the $[101,1 / 2]$ wave function. Note that the ratio of the probability of $p_{3 / 2}$ to that of $p_{1 / 2}$ in the $[101,1 / 2]$ wave function has no strong variation for $\left|\varepsilon_{\Omega}\right| \rightarrow 0$.

In order to see the effect of weakly-bound neutrons, we estimate the above $\mathrm{B}(\mathrm{E} 2)$ value using the wave functions in deformed harmonic-oscillator potentials (see, for example, Ref. [22]). Noting that

$$
\begin{aligned}
& \left\langle[220,1 / 2]\left|2 z^{2}-x^{2}-y^{2}\right|[220,1 / 2]\right\rangle=5 c_{z}^{2}-c_{\perp}^{2} \\
& \left\langle[101,1 / 2]\left|2 z^{2}-x^{2}-y^{2}\right|[101,1 / 2]\right\rangle=c_{z}^{2}-2 c_{\perp}^{2}
\end{aligned}
$$

in the unit of $\hbar / m \omega_{0}(\epsilon)$, where

$$
c_{z}^{2}=\frac{3}{3-2 \epsilon} \quad \text { and } \quad c_{\perp}^{2}=\frac{3}{3+\epsilon}
$$

and $\epsilon$ expresses the $Y_{20}$ deformation parameter of the oscillator potential, we obtain

$$
\begin{aligned}
\left\langle[220,1 / 2]\left|r^{2} Y_{20}\right|[220,1 / 2]\right\rangle & -\left\langle[101,1 / 2]\left|r^{2} Y_{20}\right|[101,1 / 2]\right\rangle \\
& =\sqrt{\frac{5}{16 \pi}}\left(4 c_{z}^{2}+c_{\perp}^{2}\right) \quad \text { in unit of } \hbar / m \omega_{0}(\epsilon) \\
& =2.62 \quad \text { in unit of } \hbar / m \omega_{0}(\epsilon) \\
& =5.66 \mathrm{fm}^{2} \quad \text { for } \mathrm{A}=12
\end{aligned}
$$

where $\epsilon=0.7$ is used and the volume conservation condition

$$
\omega_{0}(\epsilon)=\omega_{00}\left(1-\frac{\epsilon^{2}}{3}-\frac{2}{27} \epsilon^{3}\right)^{-1 / 3}
$$

is taken into account with $\hbar \omega_{00}=41 \mathrm{~A}^{-1 / 3} \mathrm{MeV}$. Using Eqs. (13) and (17) it is seen that the weakly-bound character of two neutrons in ${ }^{12} \mathrm{Be}$ makes the $\mathrm{B}\left(\mathrm{E} 2 ; I^{\pi}=K^{\pi}=0_{2}^{+} \rightarrow\right.$ $\left.I^{\pi}=2_{1}^{+}, K^{\pi}=0_{1}^{+}\right)$value larger by $(11.88 / 5.66)^{2}=4.41$. It is noted that the matrix element $\left\langle[101,1 / 2]\left|r^{2} Y_{20}\right|[101,1 / 2]\right\rangle$ in the oscillator potential, which is given in (16), changes the sign at $\epsilon=0.6$ and is positive for $\epsilon=0.7$, in agreement with the sign change of the $r^{2} Y_{20}$ matrix element of the deeply-bound [101,1/2] level shown in Fig. 1. 


\section{E0 transition in ${ }^{12} \mathrm{Be}$}

For the E0 transition, $0_{2}^{+} \rightarrow 0_{1}^{+}$, in ${ }^{12}$ Be we obtain

$$
\begin{aligned}
& \left\langle[220,1 / 2]\left|r^{2}\right|[220,1 / 2]\right\rangle=34.4 \mathrm{fm}^{2} \\
& \left\langle[101,1 / 2]\left|r^{2}\right|[101,1 / 2]\right\rangle=23.0 \mathrm{fm}^{2}
\end{aligned}
$$

by using the wave functions calculated with the potential [b] for ${ }^{12} \mathrm{Be}$. Then, using the intrinsic wave-functions, (8) and (11), we obtain

$$
\left|\left\langle 0_{1}^{+}\left|r^{2}\right| 0_{2}^{+}\right\rangle\right|=\left|\left\langle K^{\pi}=0_{1}^{+}\left|r^{2}\right| K^{\pi}=0_{2}^{+}\right\rangle\right|=11.4 \mathrm{fm}^{2}
$$

which is the neutron matrix-element. The neutron effective charge for E0 transitions, $e_{e f f}^{n}(E 0)$, may sensitively depend on both neutron excess and weak binding. The contribution to $e_{e f f}^{n}(E 0)$ coming from the part of the one-body operator obtained by subtracting the

center of mass motion is $\left(Z / A^{2}\right) e$, which is equal to $(0.028)$ e for ${ }^{12}$ Be. However, this may hardly give a reliable estimate of the actual $e_{e f f}^{n}(E 0)$ value. If the charge radius of ${ }^{12} \mathrm{Be}$ relative to that of either ${ }^{11} \mathrm{Be}$ or ${ }^{10} \mathrm{Be}$ is ever measured, it will help to obtain an estimate of the $e_{e f f}^{n}(E 0)$ value to be used in the present case [23]. Since no such measurement is presently available, writing the measured value as

$$
\left\langle 0_{2}^{+}\left|e_{e f f}^{n}(E 0) r^{2}\right| 0_{1}^{+}\right\rangle=0.87 \text { e fm } m^{2}
$$

we obtain

$$
e_{e f f}^{n}(E 0) / e=0.87 / 11.4=0.076
$$

\section{CONCLUSIONS}

We have presented the interpretation of available spectroscopic data on ${ }^{12} \mathrm{Be}$ and ${ }^{11} \mathrm{Be}$, using a model which is simple and contains the essential feature of the presence of weaklybound neutron(s) in deformed potentials. Calculated results are in good agreement with available data. We have intentionally avoided to make a quantitative comparison of spectroscopic factors with "measured" ones, partly because the presently available factors obtained from the analysis of data may, in our opinion, contain an ambiguity in the one-particle radial wave-functions used and partly because the inactive core nucleus ${ }^{10} \mathrm{Be}$ in our present 
model is different from the observed nucleus ${ }^{10} \mathrm{Be}$. The features of weakly-bound neutrons appear especially in : (a) strong E1 transitions in both ${ }^{11} \mathrm{Be}$ and ${ }^{12} \mathrm{Be}$ due to the increased and spatially-extended $s_{1 / 2}$ component in the [220,1/2] wave function; (b) The large $\mathrm{B}\left(\mathrm{E} 2 ; I^{\pi}=K^{\pi}=0_{2}^{+} \rightarrow I^{\pi}=2_{1}^{+}, K^{\pi}=0_{1}^{+}\right)$value, due to both the larger value of $\left\langle[220,1 / 2]\left|r^{2} Y_{20}\right|[220,1 / 2]\right\rangle$ and the negative value of $\left\langle[101,1 / 2]\left|r^{2} Y_{20}\right|[101,1 / 2]\right\rangle ;$ (c) The large $\left\langle[220,1 / 2]\left|r^{2}\right|[220,1 / 2]\right\rangle$ matrix element that contributes to the E0 transition, $0_{2}^{+} \rightarrow 0_{1}^{+}$.

In medium-heavy mass region one hardly finds a deformed nucleus, in which an $\Omega^{\pi}=1 / 2^{+}$ level lies close to an $\Omega^{\pi}=1 / 2^{-}$level around the ground state. Therefore, the nucleus ${ }^{11} \mathrm{Be}$ is the rare case, in which $\Omega^{\pi}=1 / 2^{+}$and $1 / 2^{-}$levels are almost degenerate and the importance of the presence of the signature-dependent term in $\mathrm{B}(\mathrm{E} 1)$ values is manifested.

One of the authors (I.H.) expresses her sincere thanks to professor Ben Mottelson for discussions.

[1] A. Bohr and B. R. Mottelson, Nuclear Structure (Benjamin, Reading, MA, 1975), Vol.II.

[2] S. Shimoura et al., submitted to Phys. Lett. B.

[3] H. Esbensen et al., Phys. Rev. C 51, 1274 (1995).

[4] A. Muta and T. Otsuka, Prog. Theor. Phys. Suppl., 142, 355 (2001).

[5] I.Ragnarsson, S. Åberg, H.-B. Håkansson and R. K. Sheline, Nucl. Phys. A361, 1 (1981).

[6] X. Li and P.-H.Heenen, Phys. Rev. C 54, 1617 (1996).

[7] T. Misu, W. Nazarewicz and S. Åberg, Nucl. Phys. A614, 44 (1997).

[8] I. Hamamoto, Phys. Rev. C 69, 041306 (2004).

[9] D. J. Millener, J. W. Olness, E. K. Warburton and S. S. Hanna, Phys. Rev. C 28, 497 (1983).

[10] N. Fukuda et al., Phys. Rev. C 70, 054606 (2004).

[11] S. D. Pain et al., Phys. Rev. Lett. 96, 032502 (2006).

[12] A. Bohr and B. R. Mottelson, Nuclear Structure (Benjamin, Reading, MA, 1969), Vol.I.

[13] I. Hamamoto, Phys. Rev. C 72, 024301 (2005).

[14] A.Navin et al., Phys. Rev. Lett. 85, 266 (2000). 
[15] W. Geithner et al., Phys. Rev. Lett. 83, 3792 (1999).

[16] H. Iwasaki et al., Phys. Lett. B481, 7 (2000).

[17] H. Iwasaki et al., Phys. Lett. B491, 8 (2000).

[18] S. Shimoura et al., Phys. Lett. B560, 31 (2003).

[19] For example, see; G. B. Hagemann, I. Hamamoto and W. Satula, Phys. Rev. C 47, 2008 (1993).

[20] H. Sagawa and K. Asahi, Phys. Rev. C 63, 064310 (2001).

[21] K. Riisager, A. S. Jensen and P. Møller, Nucl. Phys. A548, 393 (1992).

[22] J. P. Boisson and R. Piepenbring, Nucl. Phys. A168, 385 (1971).

[23] B. R. Mottelson, private communication. 
TABLE I: Calculated probabilities of $\ell_{j}$ components in the $[220,1 / 2]$ and $[101,1 / 2]$ wave functions of ${ }^{11} \mathrm{Be}$. Calculated radial wave-functions of the $\ell_{j}$ components are in general different from those of the $(n \ell j)$ wave functions which are the eigenfunctions of spherical Woods-Saxon potentials. Two parameters of a given potential, deformation $\beta$ and the depth of Woods-Saxon potentials, are adjusted so that the eigen energies of the $[220,1 / 2]$ and $[101,1 / 2]$ orbits are -0.5 and $-0.2 \mathrm{MeV}$, respectively. See the text for details.

\begin{tabular}{c|c|c|c|c|c|c|c|c|c|c}
\hline \hline \multirow{2}{*}{ orbit } & \multirow{2}{*}{ potential } & \multicolumn{7}{|c}{ probability } & \multicolumn{1}{c}{} \\
\cline { 3 - 10 } & & $s_{1 / 2}$ & $d_{3 / 2}$ & $d_{5 / 2}$ & $g_{7 / 2}$ & $g_{9 / 2}$ & $p_{1 / 2}$ & $p_{3 / 2}$ & $f_{5 / 2}$ & $f_{7 / 2}$ \\
\hline$[220,1 / 2]$ & {$[\mathrm{a}]$} & 0.51 & 0.04 & 0.44 & 0.00 & 0.01 & & & & \\
& {$[\mathrm{~b}]$} & 0.62 & 0.07 & 0.30 & 0.00 & 0.01 & & & & \\
\hline$[101,1 / 2]$ & {$[\mathrm{a}]$} & & & & & & 0.85 & 0.12 & 0.02 & 0.01 \\
& {$[\mathrm{~b}]$} & & & & & & 0.81 & 0.15 & 0.03 & 0.01 \\
\hline \hline
\end{tabular}


TABLE II: Calculated results related to the E1 transition, ground $1 / 2^{+} \rightarrow 1 / 2^{-}$at $\mathrm{Ex}=0.32 \mathrm{MeV}$, in ${ }^{11}$ Be. For simplicity, in the table $[220,1 / 2]$ and $[101,1 / 2]$ are denoted by $\left[\nu_{2}\right]$ and $\left[\nu_{1}\right]$, respectively. The value of $e_{e f f}^{n}(E 1)=(\mathrm{Z} / \mathrm{A}) \mathrm{e}$ is used. The measured value in Ref. [9] is $\mathrm{B}\left(\mathrm{E} 1 ; 1 / 2^{+} \rightarrow 1 / 2^{-}\right)=$ $(0.115 \pm 0.01) \mathrm{e}^{2} \mathrm{fm}^{2}$. See the text for details.

\begin{tabular}{c|c|c|c}
\hline \hline potential & $\begin{array}{c}\left\langle\left[\nu_{1}\right]\left|r Y_{10}\right|\left[\nu_{2}\right]\right\rangle \\
(\mathrm{fm})\end{array}$ & $\begin{array}{c}\left\langle\left[\nu_{1}\right]\left|r Y_{11}\right|\left[\nu_{2}\right]\right\rangle \\
(\mathrm{fm})\end{array}$ & $\begin{array}{c}\mathrm{B}\left(\mathrm{E} 1 ; 1 / 2^{+} \rightarrow 1 / 2^{-}\right) \\
\left(\mathrm{e}^{2} \mathrm{fm}^{2}\right)\end{array}$ \\
\hline$[\mathrm{a}]$ & -0.219 & -0.615 & $\begin{array}{c}(0.395)\left(\mathrm{e}_{\text {eff }}^{n}(\mathrm{E} 1) / \mathrm{e}\right)^{2} \\
\Rightarrow 0.052\end{array}$ \\
\hline$[\mathrm{b}]$ & -0.355 & -0.850 & $\begin{array}{c}(0.809)\left(\mathrm{e}_{\text {eff }}^{n}(\mathrm{E} 1) / \mathrm{e}\right)^{2} \\
\Rightarrow 0.107\end{array}$ \\
\hline \hline
\end{tabular}

TABLE III: Calculated results related to the E1 transition, ground $0_{1}^{+} \rightarrow 1^{-}$at $\mathrm{Ex}=2.70 \mathrm{MeV}$, in ${ }^{12} \mathrm{Be}$. The potential set [b] is used, while in the table $[220,1 / 2]$ and $[101,1 / 2]$ are denoted by $\left[\nu_{2}\right]$ and $\left[\nu_{1}\right]$, respectively. The value of $e_{\text {eff }}^{n}(E 1)=(\mathrm{Z} / \mathrm{A}) \mathrm{e}$ is used. The measured value in Ref. [17] is $\mathrm{B}\left(\mathrm{E} 1 ; 0_{1}^{+} \rightarrow 1^{-}=(0.051 \pm 0.013) \mathrm{e}^{2} \mathrm{fm}^{2}\right.$. See the text for details.

\begin{tabular}{c|c|c|c}
\hline \hline $\begin{array}{c}\left.\left\langle\nu_{1}\right]\left|r Y_{10}\right|\left[\nu_{2}\right]\right\rangle \\
(\mathrm{fm})\end{array}$ & $\begin{array}{c}\left\langle\left[\nu_{1}\right]\left|r Y_{11}\right|\left[\tilde{\nu}_{2}\right]\right\rangle \\
(\mathrm{fm})\end{array}$ & $\begin{array}{c}\mathrm{B}\left(\mathrm{E} 1 ; 0_{1}^{+} \rightarrow I^{\pi}=1^{-}, K^{\pi}=0^{-}\right) \\
\left(\mathrm{e}^{2} \mathrm{fm}^{2}\right)\end{array}$ & $\begin{array}{c}\mathrm{B}\left(\mathrm{E} 1 ; 0_{1}^{+} \rightarrow I^{\pi}=K^{\pi}=1^{-}\right) \\
\left(\mathrm{e}^{2} \mathrm{fm}^{2}\right)\end{array}$ \\
\hline-0.215 & -0.486 & $\begin{array}{c}(0.184)\left(\mathrm{e}_{e f f}^{n}(\mathrm{E} 1) / \mathrm{e}\right)^{2} \\
\Rightarrow 0.020\end{array}$ & $\begin{array}{c}(0.944)\left(\mathrm{e}_{e f f}^{n}(\mathrm{E} 1) / \mathrm{e}\right)^{2} \\
\Rightarrow 0.105\end{array}$ \\
\hline \hline
\end{tabular}




\section{Figure captions}

Figure 1 : The expectation value of $r^{2} Y_{20}$ in the [101,1/2] and [220,1/2] states as a function

of one-particle energy, $\varepsilon_{\Omega}$. For the $[220,1 / 2]$ level the $s_{1 / 2}, d_{3 / 2}, d_{5 / 2}, g_{7 / 2}$ and $g_{9 / 2}$ components are included in solving the coupled-channel equations for deformed oneparticle levels, while for the $[101,1 / 2]$ level the $p_{1 / 2}, p_{3 / 2}, f_{5 / 2}$ and $f_{7 / 2}$ components are taken into account. For comparison, two values of quadrupole deformation $\beta$ are considered. Used parameters are $r_{0}=1.25 \mathrm{fm}, a=1.0 \mathrm{fm}$ and $A=12$, while the depth of Woods-Saxon potentials is varied so that respective one-particle levels have $\varepsilon_{\Omega}$ as energy eigenvalues. 


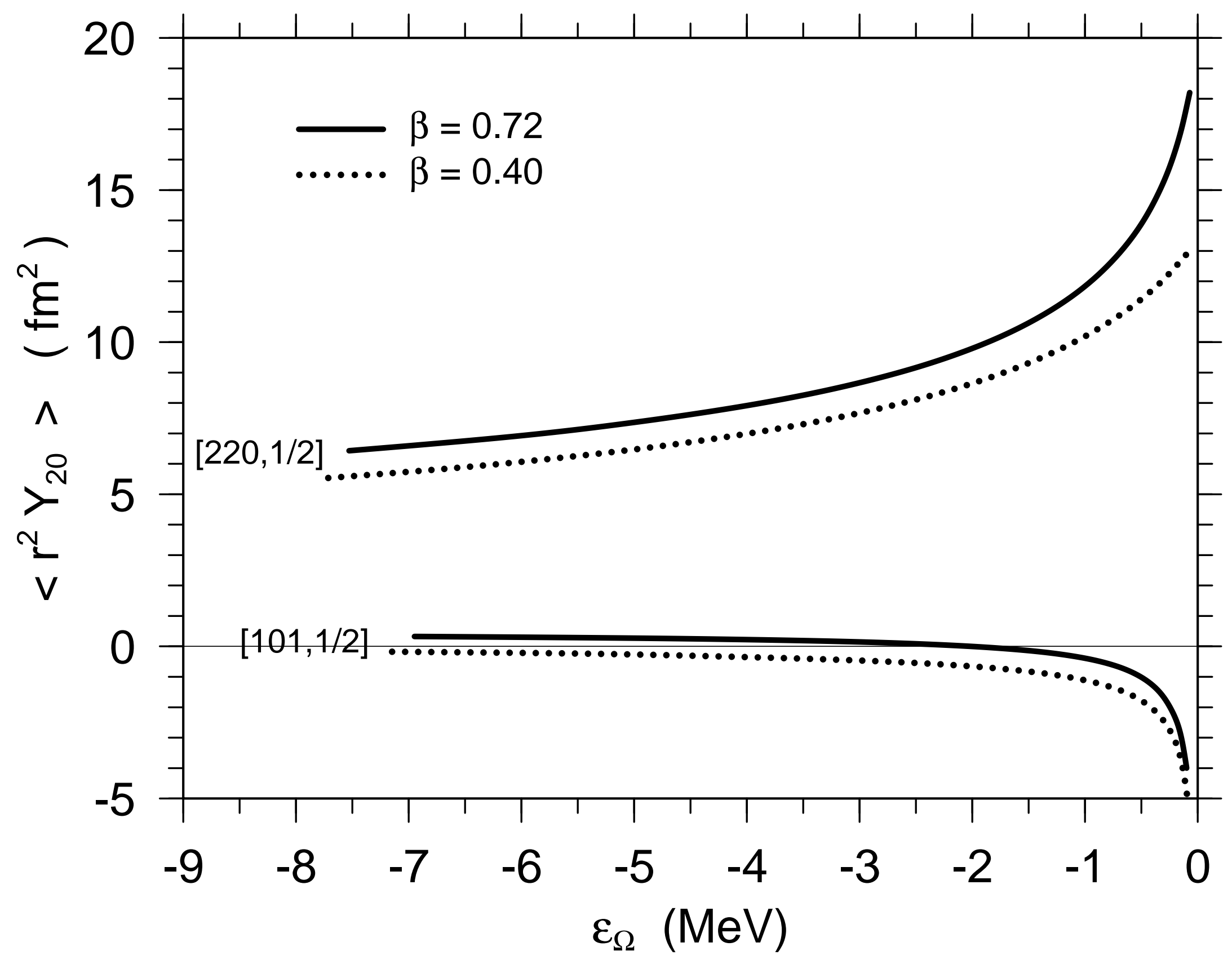

